\title{
Circumstellar effects on the Rb abundances in O-rich AGB stars ${ }^{\star}$
}

\author{
O. Zamora ${ }^{1,2}$, D. A. García-Hernández ${ }^{1,2}$, B. Plez ${ }^{3}$, and A. Manchado ${ }^{1,2,4}$ \\ 1 Instituto de Astrofísica de Canarias (IAC), 38200 La Laguna, Tenerife, Spain \\ e-mail: ozamora@iac.es \\ 2 Departamento de Astrofísica, Universidad de La Laguna (ULL), 38206 La Laguna, Tenerife, Spain \\ 3 Laboratoire Univers et Particules de Montpellier, Université Montpellier 2, CNRS, 34095 Montpellier, France \\ ${ }^{4}$ Consejo Superior de Investigaciones Científicas (CSIC), 28006 Madrid, Spain
}

Received 12 February 2014 / Accepted 10 March 2014

\section{ABSTRACT}

\begin{abstract}
For the first time we explore the circumstellar effects on the $\mathrm{Rb}$ (and $\mathrm{Zr}$ ) abundance determination in O-rich asymptotic giant branch (AGB) stars by considering the presence of a gaseous circumstellar envelope with a radial wind. A modified version of the spectral synthesis code Turbospectrum was used to deal with extended atmosphere models and velocity fields. The $\mathrm{Rb}$ and $\mathrm{Zr}$ abundances were determined from the resonant $7800 \AA \mathrm{Rb}$ I line and the $6474 \AA \mathrm{ZrO}$ bandhead, respectively, in five representative O-rich AGB stars with different expansion velocities and metallicities. By using our new dynamical models, the Rb I line profile (photospheric and circumstellar components) is very well reproduced. Interestingly, the derived Rb abundances are much lower (by 1-2 dex) in those O-rich AGB stars showing the higher circumstellar expansion velocities. The $\mathrm{Zr}$ abundances, however, remain close to the solar values. The $\mathrm{Rb}$ abundances and $[\mathrm{Rb} / \mathrm{Zr}]$ ratios derived here significantly resolve the problem of the present mismatch between the observations of intermediate-mass (4-8 $M_{\odot}$ ) Rb-rich AGB stars and the AGB nucleosynthesis theoretical predictions.
\end{abstract}

Key words. stars: AGB and post-AGB - stars: abundances - stars: evolution - nuclear reactions, nucleosynthesis, abundances stars: atmospheres - stars: late-type

\section{Introduction}

The asymptotic giant branch (AGB) is the last nuclear-burning phase of low- and intermediate-mass stars $\left(0.8 \leq M \leq 8 M_{\odot}\right)$. The AGB stars are among the main contributors to the chemical enrichment of the interstellar medium since they suffer strong mass loss together with nucleosynthesis processes (see e.g. Busso et al. 1999). Low-mass AGB stars $\left(M<4 M_{\odot}\right)$ can turn $\mathrm{C}$-rich (i.e. $\mathrm{C} / \mathrm{O}>1$ ) because of the dredge-up of carbon from the bottom of the convective envelope to the stellar surface. The $s$-process allows the production of elements heavier than iron by slow neutron-captures. In these stars, the ${ }^{13} \mathrm{C}(\alpha, \mathrm{n}){ }^{16} \mathrm{O}$ reaction is assumed to operate as the main neutron source (e.g. Abia et al. 2001). On the other hand, intermediate-mass AGB stars $\left(4 \leq M \leq 8 M_{\odot}\right)$ are O-rich stars $(\mathrm{C} / \mathrm{O}<1)$ because of the operation of hot bottom burning (HBB), which burns carbon at the base of the convective envelope, thus preventing the formation of a carbon star (Sackmann \& Boothroyd 1992). In these carbon stars, the $s$-process elements are expected to form mainly by the neutrons released by the ${ }^{22} \mathrm{Ne}(\alpha, \mathrm{n})^{25} \mathrm{Mg}$ reaction, in a higher neutron density environment than in lower mass AGB stars (García-Hernández et al. 2006). The relative abundance of s-elements such as $\mathrm{Rb}$ to other neighboring ones such as $\mathrm{Sr}, \mathrm{Y}$, and $\mathrm{Zr}$ is an indicator of the neutron density, namely a discriminant of the stellar mass and the main neutron source at the s-process site (Lambert et al. 1995; Abia et al. 2001; García-Hernández et al. 2006).

Observationally, a low $[\mathrm{Rb} / \mathrm{Zr}]$ ratio $(<0)$ is found in lowmass AGB stars (Plez et al. 1993; Lambert et al. 1995; Abia et al. 2001) while higher mass AGB stars display $[\mathrm{Rb} / \mathrm{Zr}]>0$

\footnotetext{
* Appendices are available in electronic form at http://www . aanda.org
}

(García-Hernández et al. 2006, 2007, 2009). García-Hernández et al. (2006, 2009, hereafter Paper I and Paper II, respectively) derived the $\mathrm{Rb}$ and $\mathrm{Zr}$ abundances in several Galactic and Magellanic Cloud intermediate-mass AGB stars among a sample of $\mathrm{OH} / \mathrm{IR}$ stars. The $\mathrm{Rb}$ abundances and $[\mathrm{Rb} / \mathrm{Zr}]$ ratios found in these objects represent a challenge for theoretical AGB nucleosynthesis models, which do not predict the extreme Rb overabundances $([\mathrm{Rb} / \mathrm{Fe}] \gtrsim 2$ dex) and extraordinarily high $[\mathrm{Rb} / \mathrm{Zr}]$ ratios observed (van Raai et al. 2012; Karakas et al. 2012). However, the $\mathrm{Rb}$ abundance was derived from the resonant $\mathrm{Rb} \mathrm{I}$ absorption line at $7800 \AA$, using hydrostatic model atmospheres. The $\mathrm{Rb}$ I line is probably affected by contamination from one or more circumstellar (CS) components, as has already been suggested by the detection of blue-shifted CS Rb I absorption lines in several of these extreme O-rich AGB stars (see Papers I and II).

In the present Letter, we explore for the first time the CS effects on the $\mathrm{Rb}$ and $\mathrm{Zr}$ abundances derived in extreme O-rich AGB stars. To this end, we use more realistic model atmospheres that include a gaseous CS envelope. The much lower $\mathrm{Rb}$ abundances (and $[\mathrm{Rb} / \mathrm{Zr}]$ ratios) derived here significantly alleviate the actual mismatch between the AGB nucleosynthesis predictions and the optical observations of intermediate-mass (4-8 $M_{\odot}$ ) Rb-rich AGB stars.

\section{The sample stars}

We selected a representative sample of five massive Rb-rich AGB stars from Papers I and II: four Galactic stars and one LMC star, covering several $\mathrm{OH}$ expansion velocities, variability periods, and metallicities The relevant information for the sample stars is shown in Table A.1. All stars belong to the 
$\mathrm{OH} / \mathrm{IR}$ class, with $\mathrm{OH}$ expansion velocities and variability periods available in the literature. We chose those stars with high signal-to-noise ( $S / N \geq 30-50)$ optical spectra for reliable $\mathrm{Rb}$ and $\mathrm{Zr}$ abundance determinations. The resolving power of the observations is $R=\lambda / \Delta \lambda=400000$ to 50000 for the Galatic stars and $R \sim 60000$ for the LMC star (see Papers I and II for further observational details). The sample stars display a variety of Rb I $7800 \AA$ line strengths and profiles. Two stars (IRAS 05098-6422 and IRAS 18429-1721) display weak nonshifted $\mathrm{Rb}$ I lines while the other three stars (IRAS 06300+6058, IRAS 19059-2219, and IRAS 04498-6842) show a blueshifted $\mathrm{Rb}$ I absorption feature that is indicative of a CS envelope moving outwards in the line of sight ${ }^{1}$. As was already shown in Papers I and II, classical MARCS hydrostatic models are not able to account for the blue-shifted absorption feature. Furthermore, the Rb abundances derived in Papers I and II with these hydrostatic model atmospheres are significantly larger than AGB nucleosynthesis models predictions (see e.g. van Raai et al. 2012). The most remarkable case is the LMC star IRAS 04498-6842 with $[\mathrm{Rb} / \mathrm{M}]=+5.0^{2}$.

\section{Abundance analysis using dynamical models}

\subsection{Turbospectrum spectral synthesis code with extended atmospheres and velocity fields}

For a proper analysis of the circumstellar component around the $\mathrm{Rb}$ I 7800 A line, we have modified the v12.1 of the spectral synthesis code Turbospectrum (Alvarez \& Plez 1998; Plez 2012) to deal with extended atmospheres and velocity fields. The Doppler effect due to the expanding envelope of the star is implemented in Turbospectrum by the modification of the routines that compute the line intensities at the stellar surface. Concerning the radiative transfer, the source function is assumed to be the same as computed in the static case without a wind (see Gustafsson et al. 2008). The scattering term of the source function $\left(\propto \sigma_{\lambda} J_{\lambda}\right)$ is not shifted to save computing time. Scattering is only included for the continuum. We carefully checked this approximation by comparing it with Monte Carlo simulations (see Sect. 3.3) because in cool CS envelopes photons are mostly scattered in resonance lines and are not thermally emitted as we assume here. In this way, the scattering term is computed as in the static case using the Feautrier method (Nordlund 1984; Gustafsson et al. 2008). The velocity field is taken into account through a shift of the absorption coefficient $\kappa_{\lambda}$. The source function is built using the static $\sigma_{\lambda} J_{\lambda}$ and the shifted $\kappa_{\lambda} B_{\lambda}$. The emerging intensity is then computed in the observer frame by a direct quadrature of the source function. This algorithm is sensitive to numerical errors (Mihalas \& Kunasz 1986). Therefore, a high sampling of the optical depth and the wavelength points is used to minimise errors in the opacity interpolation.

\subsection{Dynamical atmosphere models}

The atmospheric parameters adopted for the individual analysis of each star are taken from Papers I and II, using solar reference abundances by Grevesse et al. (2007). Furthermore, our

\footnotetext{
1 The observed $\mathrm{Rb}$ I line in IRAS $06300+6058$ is resolved in two components (circumstellar and stellar).

2 In Paper II, an average metallicity of $[\mathrm{M} / \mathrm{H}]=-1.4$, as estimated from a few very weak metallic lines around the Rb I line, was used in the synthesis. Here, however, we prefer to adopt the LMC average metallicity $([\mathrm{M} / \mathrm{H}]=-0.3)$ in the synthesis in order to be consistent with the original metallicity of the atmosphere model.
}

dynamical models are constructed from the original MARCS hydrostatic atmosphere model structure, expanding the atmosphere radius by the inclusion of a wind out to $\sim 5$ stellar radii, with a radial velocity field in spherical symmetry. The stellar radius $R_{*}$ is defined as the radius corresponding to $r\left(\tau_{\text {Ross }}=1\right)$ in the MARCS hydrostatic model, where $r$ is the distance from the stellar centre and $\tau_{\text {Ross }}$ the Rosseland optical depth. The stellar wind is computed under the assumptions of mass conservation (Eq. (1)) and radiative thermal equilibrium (Eq. (2)), following a classical $\beta$-velocity law (Eq. (3)),

$$
\begin{aligned}
& \rho(r)=\frac{\dot{M}}{4 \pi r^{2} v(r)} \\
& r T^{2}=\text { constant }=r_{\text {out }} T_{\text {out }}^{2} \\
& v(r)=v_{0}+\left(v_{\infty}-v_{0}\right)\left(1-\frac{R_{*}}{r}\right)^{\beta},
\end{aligned}
$$

where $\rho(r)$ is the density of the envelope at radius $r, \dot{M}$ is the mass-loss rate, and $v(r)$ is the velocity of the envelope. The velocity $v(r)$ is calculated by means of Eq. (3), where $v_{0}$ is a reference velocity for the onset of the wind and the $\beta$ exponent is an arbitrary free parameter. For the onset of the wind, we take $v_{0}=v\left(R_{*}\right)$ and the extension of the envelope begins from the outer radius of the hydrostatic model. The envelope is extended making use of Eq. (2), layer by layer, out to $r_{\max }$. The distance $r_{\max }$ corresponds to the maximum radius in our calculations, with temperature $T_{\max }=900 \mathrm{~K}$, because Turbospectrum cannot compute for lower temperatures because of numerical reasons. For the mass-loss rate $\dot{M}$ and the $\beta$ exponent, we use values in the typical range of AGB stars: $\dot{M} \sim 10^{-9}-10^{-4} M_{\odot} \mathrm{yr}^{-1}$ in steps of factors of 10 and $\beta \sim 0-1.6$ in steps of 0.2 . Finally, we assume $v_{\text {exp }}(\mathrm{OH})$ as the terminal velocity $v_{\infty}$ (see Table A.1) because the $\mathrm{OH}$ maser emission is found at very large distances of the central star (see e.g. Decin et al. 2010). Figure A.1 shows examples of the $\beta$-velocity law adopted in our dynamical models.

The resulting grid of synthetic spectra is compared to the observed spectra in order to find the best fit to the $\mathrm{Rb}$ I line profile and the adjacent pseudocontinuum. Figure A.2 shows several fits for the Rb I $7800 \AA$ and K I $7699 \AA$ lines (with similar atomic parameters) in IRAS $06300+6058$ to check the consistency of our models. In the left panel, we modify the $(\dot{M}, \beta)$ pair and the abundance of $\mathrm{Rb}$ until the best fit (in red) to the observations (in black) is obtained. Remarkably, the best model $(\dot{M}, \beta)=\left(1 \times 10^{-7}, 0.2\right)$ gives a $\mathrm{Rb}$ abundance of +0.5 , which is much lower than the hydrostatical abundance (see Table A.1). Curiously, the CS K I component is blue-shifted by $\sim 2-3 \mathrm{~km} \mathrm{~s}^{-1}$ relative to that in $\mathrm{Rb} \mathrm{I}$, suggesting a slightly higher velocity for the K I expanding gas. Thus, we test the same models for the $\mathrm{K} I$ line fitting in the right panel but varying the $\mathrm{K}$ abundance and the terminal velocity. Acceptable fits to the K I line are obtained for solar $\mathrm{K}$ abundances and a slightly higher terminal velocity of $\sim 15 \mathrm{~km} \mathrm{~s}^{-1}$, as suggested by the blue-shifted CS K I line. We note that increasing the $\mathrm{K}$ abundance may improve the fit a little bit. At present we cannot discard some $\mathrm{K}$ production in these stars as the consequence of proton-captures on Ar nuclei during HBB (Ventura et al. 2012).

\subsection{Monte Carlo simulations}

As we already commented in Sect. 3.1, to check if our neglect of line scattering in Turbospectrum does not affect the interpretation of the observed spectra, we performed complementary Monte Carlo simulations (MCS) that only take into account photon scattering for the radiative transfer. Three-dimensional MCS 
O. Zamora et al.: Circumstellar effects on the Rb abundances in O-rich AGB stars

Table 1. Atmosphere parameters and abundances derived using dynamical models vs. hydrostatic models.

\begin{tabular}{cccccc|cccc}
\hline \hline IRAS name & $T_{\text {eff }}(\mathrm{K})$ & $\log g$ & $\beta$ & $\dot{M}\left(M_{\odot} \mathrm{yr}^{-1}\right)$ & $v\left(\mathrm{~km} \mathrm{~s}^{-1}\right)$ & {$[\mathrm{Rb} / \mathrm{M}]_{\text {static }}^{\text {ref }}{ }^{a}$} & {$[\mathrm{Rb} / \mathrm{M}]_{\text {static }^{b}}$} & {$[\mathrm{Rb} / \mathrm{M}]_{\mathrm{dyn}}{ }^{b}$} & {$[\mathrm{Zr} / \mathrm{M}]_{\mathrm{dyn}}{ }^{b}$} \\
\hline $05098-6422$ & 3000 & -0.5 & 1.0 & $1.0 \times 10^{-8}$ & 6 & 0.1 & $0.0 \pm 0.4$ & $0.0 \pm 0.4$ & $\leq 0.3 \pm 0.3$ \\
$06300+6058$ & 3000 & -0.5 & 0.2 & $1.0 \times 10^{-7}$ & 12 & 1.6 & $1.9 \pm 0.4$ & $0.5 \pm 0.7$ & $\leq 0.1 \pm 0.3$ \\
$18429-1721$ & 3000 & -0.5 & 1.0 & $1.0 \times 10^{-8}$ & 7 & 1.2 & $1.2 \pm 0.4$ & $1.0 \pm 0.4$ & $\leq 0.3 \pm 0.3$ \\
$19059-2219$ & 3000 & -0.5 & 0.4 & $1.0 \times 10^{-7}$ & 13 & $2.3 / 2.6$ & $2.4 \pm 0.4$ & $0.8 \pm 0.7$ & $\leq 0.3 \pm 0.3$ \\
\hline \multicolumn{7}{c}{ LMC star } \\
\hline $04498-6842$ & 3400 & 0.0 & 1.0 & $1.0 \times 10^{-7}$ & 13 & $3.9^{c}$ & $3.3 \pm 0.4$ & $1.5 \pm 0.7$ & $\leq 0.3 \pm 0.3$ \\
\hline
\end{tabular}

Notes. ${ }^{(a)}$ See Papers I and II. ${ }^{(b)}$ The uncertainties represent the formal errors due to the sensitivity of the derived abundances to slight changes in the model atmosphere parameters $\left(\Delta T_{\text {eff }}= \pm 100 \mathrm{~K}, \Delta[\mathrm{M} / \mathrm{H}]= \pm 0.3, \Delta \xi= \pm 1 \mathrm{~km} \mathrm{~s}^{-1}, \Delta \log g=+0.5, \Delta F W H M=50 \mathrm{~m} \AA, \Delta \beta=0.2\right.$, $\left.\Delta \log \left(\dot{M} / M_{\odot} \mathrm{yr}^{-1}\right)=1.0\right)$ for each star. ${ }^{(c)}$ We scale the $\mathrm{Rb}$ overabundance derived by Paper II, $[\mathrm{Rb} / \mathrm{M}]=+5.0$, to the adopted LMC metallicity $[\mathrm{M} / \mathrm{H}]=-0.3$.

were carried out throwing a large number of photons $\left(\sim 10^{6}\right.$ for accurate statistics) through the expanding envelope of a star, taking the initial wavelength, optical depth at which the photon will be scattered $\tau_{\lambda}$, and throwing direction of the incident photon as random numbers. The photon of wavelength $\lambda$ has an absorption probability $1-\mathrm{e}^{-\tau_{\lambda}}$. The random $\tau_{\lambda}$ at which the photon will be scattered follows uniform statistics, i.e. the same number of photons are thrown for each wavelength in all directions. Thus, we study the emergent photon distribution (spectrum) obtained for the CS component around the K I $7699 \AA$ A line. The results can be extrapolated to the CS component of the Rb I $7800 \AA$ line, taking into account that the density of $\mathrm{K}$ is $\sim 300$ times the $\mathrm{Rb}$ density for solar composition. As we only want to study how the line profile changes qualitatively with a very simple code the parameters describing the envelope are assumed constant in each simulation: temperature, $T(\mathrm{~K})$; density of $\mathrm{K} \mathrm{I}$ atoms, $n\left(\mathrm{~cm}^{-3}\right)$; expansion velocity, $v_{\exp }\left(\mathrm{km} \mathrm{s}^{-1}\right)$; and maximum envelope size, $R_{\max }\left(\mathrm{cm}, 1 \mathrm{AU} \sim 1.5 \times 10^{10} \mathrm{~cm}\right)$, We performed 216 simulations in total, covering the following space of parameters: $T=10$, $100,1000 \mathrm{~K} ; n=1 \times 10^{-6}, 1 \times 10^{-5}, 1 \times 10^{-4}, 1 \times 10^{-3} \mathrm{~cm}^{-3}$; $v_{\exp }=5,10,15 \mathrm{~km} \mathrm{~s}^{-1}$ and $R_{\max }=1 \times 10^{13}, 1 \times 10^{14}, 1 \times 10^{15}$, $1 \times 10^{16}, 1 \times 10^{17}, 1 \times 10^{18} \mathrm{~cm}$. A representative subset of the results obtained for $T=1000 \mathrm{~K}$ - the coolest temperature that Turbospectrum is able to compute - and $v_{\exp }=10 \mathrm{~km} \mathrm{~s}^{-1}$ are shown in Fig. B.1. For other envelope temperatures or expansion velocities, the resulting line profiles are very similar. In short, the most frequent line profile obtained from MCS is a blue-shifted absorption feature with a weak and broader emission component. The envelope parameters of the simulations displaying a blue-shifted absorption feature with weak emission are within the range of the dynamical atmosphere models used in Turbospectrum, which ends at $R_{\max }=1.6 \times 10^{14} \mathrm{~cm}$. For example, for $R_{\max }=1.6 \times 10^{14} \mathrm{~cm}$ some weak emission is obtained in two MCS only, corresponding to a higher density of $n=1 \times 10^{-3} \mathrm{~cm}^{-3}$. PCygni profiles, i.e. with a strong emission component, are less frequent (14\%) and they are obtained only at very high densities and/or large envelope sizes. In the rest (30\%) of the MCS, the spectrum is flat and no absorption and/or emission line is found in the distribution of the emergent photons for very low density $\left(n \leq 1 \times 10^{-5} \mathrm{~cm}^{-3}\right)$ and small envelope size $\left(R_{\max }<10^{15} \mathrm{~cm}\right)$ values (Fig. B.1).

The optical observations of $\mathrm{Rb}$ in massive Galactic AGB stars tell us that a non-shifted (purely photospheric) or a blueshifted Rb I (with a CS component) line are by far the most frequent cases (see Paper I). There is only one star IRAS 12377-6102 with a clear PCygni Rb I profile; perhaps the star IRAS 18050-2213 also shows some weak emission. These two stars are among the reddest Rb-rich AGB stars, also showing very high $\mathrm{OH}$ expansion velocities $\left(\sim 20 \mathrm{~km} \mathrm{~s}^{-1}\right)$, and they are presumably the most evolved and extreme stars. Indeed, Justtanont et al. (2013) estimate envelope sizes from $\sim 9 \times$ $10^{15} \mathrm{~cm}$ to $2.5 \times 10^{16} \mathrm{~cm}$ in a few Galactic OH/IR stars (massive AGBs) from Herschel observations. Only one star in this work, WX Psc, has an optical counterpart. Although the $\mathrm{S} / \mathrm{N}$ around $7800 \AA$ is quite low, a strong Rb I absorption line is clearly detected in the WX Psc optical spectrum (Paper I). Interestingly, the estimated mass-loss rate for WX Psc $\left(\sim 10^{-5} M_{\odot} \mathrm{yr}^{-1}\right)$ is lower by one order of magnitude than that for the more obscured stars. Furthermore, Betelgeuse observations (Plez \& Lambert 2002) show that a mass-loss rate of $\sim 10^{-6} M_{\odot} \mathrm{yr}^{-1}$ results in a double K I $7699 \AA$ line with emission in the center thanks to the photon scattering but this emission is feeble in the integrated star spectrum and the circumstellar absorption dominates. Taking into account the results of the MCS and the cited observational constraints, we conclude that Turbospectrum (Sect. 3.1), provides a reasonable approximation for the stars studied here.

\section{Results and discussion}

The parameters of the dynamical atmosphere models providing the best fit to the observations and the derived $\mathrm{Rb}$ and $\mathrm{Zr}$ abundances $\left([\mathrm{Rb} / \mathrm{M}]_{\text {dyn }}\right.$ and $\left.[\mathrm{Zr} / \mathrm{M}]_{\text {dyn }}\right)$ are shown in Table 1 . The $\mathrm{Rb}$ abundances $\left([\mathrm{Rb} / \mathrm{M}]_{\text {static }}^{\text {ref }}\right)$ as determined in Papers I and II from hydrostatic models are also shown for comparison. We note, however, that Papers I and II used solar abundances from Grevesse \& Sauval (1998) while we assume here the most recent solar composition by Grevesse et al. (2007). For this reason, Table 1 also lists the re-derived $\mathrm{Rb}$ abundances $\left([\mathrm{Rb} / \mathrm{M}]_{\text {static }}\right)$ using the hydrostatic models with our adopted solar abundances. Our static Rb abundances agree well, within the errors, with those previously derived in Papers I and II.

Interestingly, the new $\mathrm{Rb}$ abundances (in the range $[\mathrm{Rb} / \mathrm{M}] \sim$ 0.0-1.5 dex; Table 1) derived from our dynamical models display a dramatic decrease of $1.4 \mathrm{dex}$ to $1.8 \mathrm{dex}$ with respect to the static case (Table 1) in the more extreme stars, namely those stars showing the highest expansion velocities and massloss rates such as IRAS 06300+6058, IRAS 19059-2219, and IRAS 04498-6842. However, for the less extreme stars with a lower expansion velocity and mass-loss rate (IRAS 05098-6422 and IRAS 18429-1721), the Rb abundances obtained from dynamical models remain close, within 0.2 dex, to those from hydrostatic models. In Fig. 1, we display the observed Rb I line profiles in our O-rich AGB sample (black dots) together with the best synthetic spectra as obtained from the new dynamical 


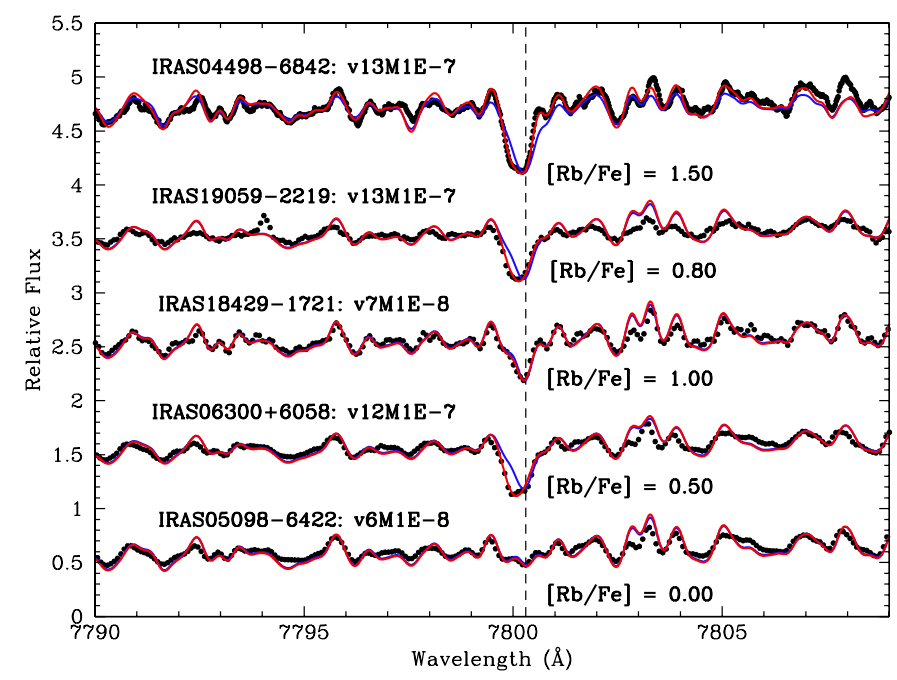

Fig. 1. $\mathrm{Rb}$ abundances derived in the sample stars using dynamical models. The location of the Rb I stellar line is indicated by a dashed line. Dynamical models providing the best fits to the observations (black dots) are indicated by a red line. Hydrostatic models are also shown for comparison (blue lines). The expansion velocity and the mass-loss rate adopted in the models also are indicated for each star.

models (red lines) versus the static ones (blue lines). Our dynamical atmosphere models reproduce the observed $\mathrm{Rb}$ I line profiles (photospheric and circumstellar components) very well, much better than the classical hydrostatic models. The $6474 \AA$ $\mathrm{ZrO}$ bandhead, because it is formed deeper in the atmosphere, is less affected than the $\mathrm{Rb} \mathrm{I}$ line, and the $\mathrm{Zr}$ abundances (nearlysolar; see Fig. A.3) derived from dynamical models are similar to those obtained with the hydrostatic models (see Papers I and II).

Remarkably, we find that relatively low mass-loss rates $\left(\sim 10^{-7}-10^{-8} M_{\odot} \mathrm{yr}^{-1}\right)$ give superior fits to the observed $\mathrm{Rb} \mathrm{I}$ line profiles. Higher mass-loss rates of $\geq 10^{-6} M_{\odot} \mathrm{yr}^{-1}$ give $\mathrm{Rb} \mathrm{I}$ absorption lines that are too strong even for solar Rb abundances (see Fig. A.2). According to Justtanont et al. (2013), the envelope size of $\sim 1 \times 10^{16} \mathrm{~cm}$ and the mass-loss rate of $\sim 10^{-4} M_{\odot} \mathrm{yr}^{-1}$ estimated in optically obscured OH/IR AGB stars can be taken as upper limits for massive AGB stars with optical counterparts; i.e. like our sample stars with useful spectra around the $\mathrm{Rb}$ I line because the heavily obscured OH/IR massive AGB stars studied by Justtanont et al. (2013) have already entered the superwind phase. Our best fits for the Rb I $7800 \AA$ line profiles in optically bright massive AGB stars are those with mass-loss rates lower than $\sim 10^{-6} M_{\odot} \mathrm{yr}^{-1}$. Thus, we conclude that the $\mathrm{Rb}$ I line profiles observed in massive AGB stars are consistent with these stars still experiencing relatively low massloss rates just before the superwind phase when the $\mathrm{Rb}$ density and the envelope size are not very high and this is supported by the MCS presented above (Sect. 3.3).

Standard nucleosynthesis models for intermediate-mass AGB stars show that the predicted $\mathrm{Rb}$ abundances range from $[\mathrm{Rb} / \mathrm{M}] \sim 0.0$ up to $1.44 \mathrm{dex}$, depending on the progenitor mass and metallicity (see van Raai et al. 2012); the predicted $\mathrm{Rb}$ production increases with increasing stellar mass and decreasing metallicity. Maximum [Rb/M] overabundances of 1.04 and 1.44 are found for a solar metallicity $6.5 M_{\odot}$ star and for a LMC metallicity $6 M_{\odot}$ star, respectively (van Raai et al. 2012).
More recently, Karakas et al. (2012) have delayed the beginning of the superwind phase in solar metallicity nucleosynthesis models of massive AGB stars. These models produce more $\mathrm{Rb}$ than in the standard van Raai et al. models because the star experiences more thermal pulses before the superwind phase at the very end of the AGB. The maximum $\mathrm{Rb}$ production $([\mathrm{Rb} / \mathrm{M}]=$ 1.34 dex) is predicted to occur for the $6 M_{\odot}$ case. By considering the error bars in the spectroscopic analysis (see Table 1) and the theoretical uncertainties (see e.g. van Raai et al. 2012; Karakas et al. 2012), the Rb abundances are now in fair agreement with the massive AGB nucleosynthesis models, both standard and with delayed superwinds. The nearly-solar derived $\mathrm{Zr}$ abundances in IRAS 05098-6422, IRAS 06300+6058, and IRAS 19059-2219 (Table 1) translate into $[\mathrm{Rb} / \mathrm{Zr}]$ ratios of $-0.3,0.4$, and 0.5 , respectively, which agree quite well with the theoretical predictions $(-0.2 \leq[\mathrm{Rb} / \mathrm{Zr}]<0.6)$. However, the $[\mathrm{Rb} / \mathrm{Zr}]$ ratios in IRAS 18429-1721 (0.7) and IRAS 04498-6842 (1.2) are still higher than predicted. As already pointed out in the literature (see e.g. van Raai et al. 2012), a possible solution to this observational problem is that gaseous $\mathrm{Zr}$, with a condensation temperature $(1741 \mathrm{~K})$ higher than that for $\mathrm{Rb}(800 \mathrm{~K})$, condensates into dust grains, producing the apparent $\mathrm{Zr}$ underabundance that we measure from the $\mathrm{ZrO}$ bandheads.

In summary, the $\mathrm{Rb}$ abundances and $[\mathrm{Rb} / \mathrm{Zr}]$ ratios derived here significantly resolve the problem of the present mismatch between the observations of massive (4-8 $M_{\odot}$ ) Rb-rich AGB stars and the theoretical predictions. In the near future, we plan to carry out a chemical analysis based on these new dynamical models for all the Rb-rich AGB stars already studied in Papers I and II. This undoubtedly will help us to constrain the actual nucleosynthesis models for the more massive AGB stars.

Acknowledgements. O.Z., D.A.G.H. and A.M. acknowledge support provided by the Spanish Ministry of Economy and Competitiveness under grant AYA2011-27754. We acknowledge the anonymous referee and N. Kameswara Rao for comments that helped to improve the paper.

\section{References}

Abia, C., Busso, M., Gallino, R., et al. 2001, ApJ, 559, 1117

Alvarez, R., \& Plez, B. 1998, A\&A, 330, 1109

Busso, M., Gallino, R., \& Wasserburg, G. J. 1999, ARA\&A, 37, 239

Decin, L., Justtanont, K., De Beck, E., et al. 2010, A\&A, 521, L4

García-Hernández, D. A., García-Lario, P., Plez, B., et al. 2006, Science, 314, 1751

García-Hernández, D. A., García-Lario, P., Plez, B., et al. 2007, A\&A, 462, 711 García-Hernández, D. A., Manchado, A., Lambert, D. L., et al. 2009, ApJ, 705, L31

Grevesse, N., \& Sauval, A. J. 1998, Space Sci. Rev., 85, 161

Grevesse, N., Asplund, M., \& Sauval, A. J. 2007, Space Sci. Rev., 130, 105 Gustafsson, B., Edvardsson, B., Eriksson, K., et al. 2008, A\&A, 486, 951

Justtanont, K., Teyssier, D., Barlow, M. J., et al. 2013, A\&A, 556, A101

Karakas, A. I., García-Hernández, D. A., \& Lugaro, M. 2012, ApJ, 751, 8

Lambert, D. L., Smith, V. V., Busso, M., Gallino, R., \& Straniero, O. 1995, ApJ, 450,302

Mihalas, D., \& Kunasz, P. B. 1986, J. Comput. Phys., 64, 1

Nordlund, A. 1984, in Methods in Radiative Transfer, ed. W. Kalkofen (Cambridge, New York: Cambridge University Press), 211

Plez, B. 2012, Turbospectrum: Code for spectral synthesis, Astrophysics Source Code Library 1205.004

Plez, B., \& Lambert, D. L. 2002, A\&A, 386, 1009

Plez, B., Smith, V. V., \& Lambert, D. L. 1993, ApJ, 418, 812

Sackmann, I.-J., \& Boothroyd, A. I. 1992, ApJ, 392, L71

van Raai, M. A., Lugaro, M., Karakas, A. I., García-Hernández, D. A., \& Yong, D. 2012, A\&A, 540, A44

Ventura, P., D’Antona, F., Di Criscienzo, M., et al. 2012, ApJ, 761, L30

Pages 5 to 6 are available in the electronic edition of the journal at http: //www . aanda. org 


\section{Appendix A: Additional table and figures}

Table A.1. The sample stars.

\begin{tabular}{cccc}
\hline \hline IRAS name & SpType & $v_{\text {exp }}(\mathrm{OH})\left(\mathrm{km} \mathrm{s}^{-1}\right)$ & Period (days) \\
\hline \multicolumn{4}{c}{ Galactic stars } \\
\hline $05098-6422$ & $\mathrm{M} 7 \mathrm{e}$ & 6.0 & 394 \\
$06300+6058$ & $\mathrm{M} 7+$ & 12.3 & 440 \\
$18429-1721$ & $\mathrm{M} 9$ & 6.9 & 1508 \\
$19059-2219$ & $\mathrm{M} 8$ & 13.3 & 510 \\
\hline \multicolumn{4}{c}{ LMC star } \\
\hline $04498-6842$ & $\mathrm{M}$ & 13.0 \\
\hline
\end{tabular}

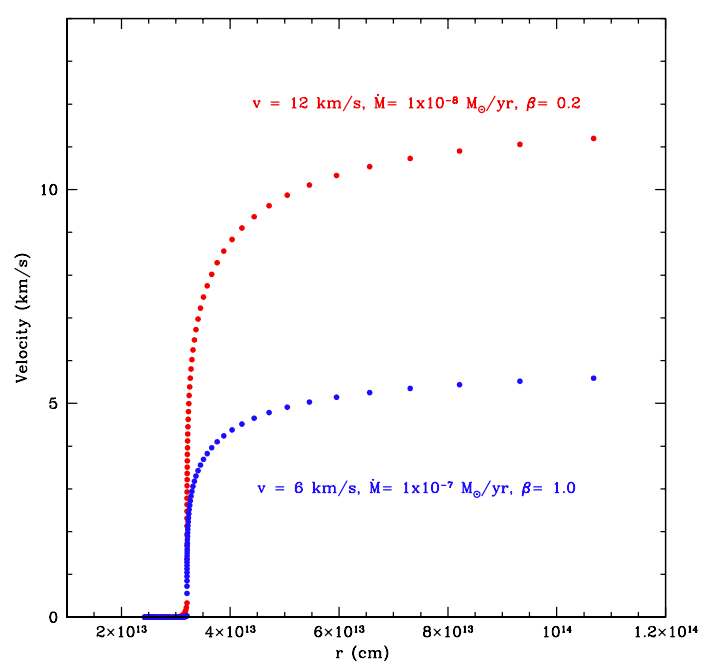

Fig. A.1. Velocity law (velocity vs. distance from the star) in two of our AGB wind models. Both models are based on the MARCS hydrostatic model with $T_{\text {eff }}=3500 \mathrm{~K}, \log g=-0.5$, and solar chemical composition. The red model is computed with velocity at infinite of $12 \mathrm{~km} \mathrm{~s}^{-1}$, mass-loss rate of $10^{-8} M_{\odot} \mathrm{yr}^{-1}$, and $\beta$ exponent of 0.2 . The blue model has a velocity at infinite of $6 \mathrm{~km} \mathrm{~s}^{-1}$, mass-loss rate of $10^{-7} M_{\odot} \mathrm{yr}^{-1}$, and $\beta$ exponent of 1.0. We note that a smaller step is taken where the velocity gradient is steepest.
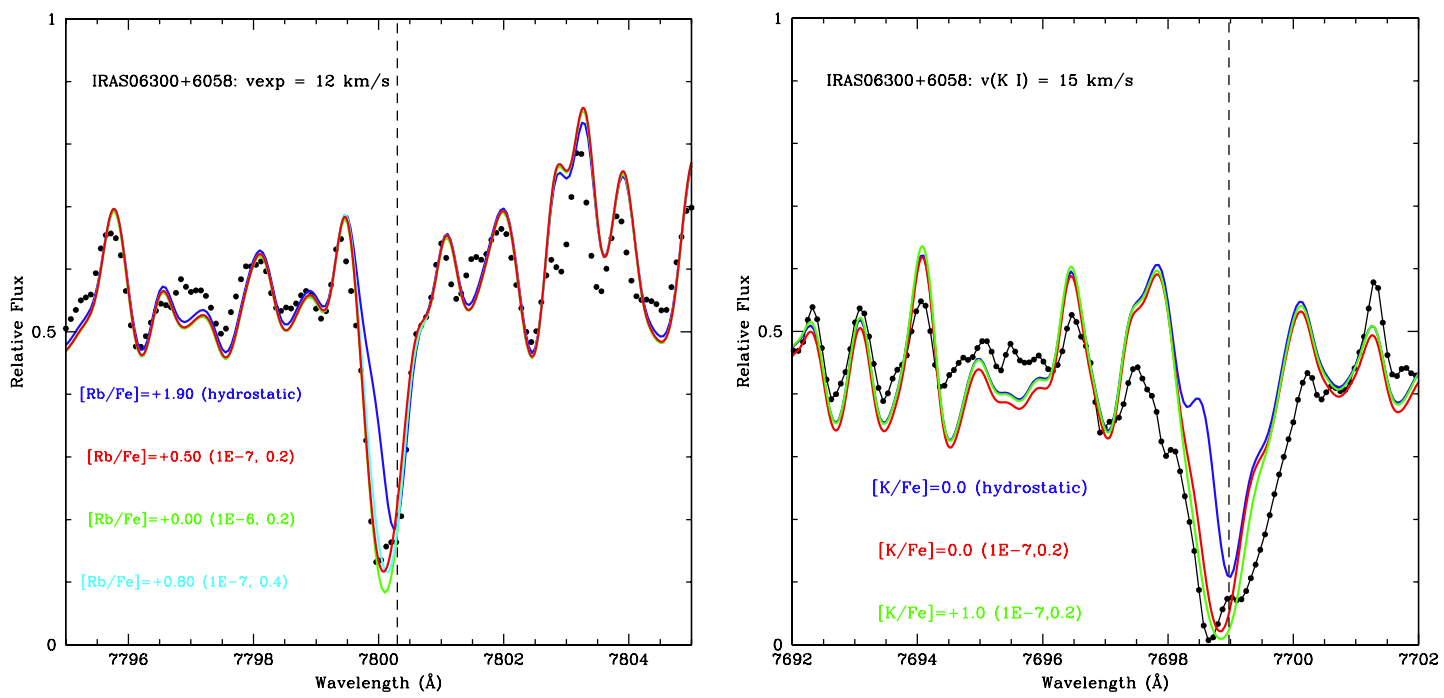

Fig. A.2. Rb I (left panel) and K I (right panel) line profiles obtained using dynamical models given by the $(\dot{M}, \beta)=\left(1 \times 10^{-7}, 0.2\right)$ pair $($ see Sect. 3.2) for the star IRAS $06300+6058$. The hydrostatic model (blue line) is also shown for comparison. The colours have similar meaning in the two panels but in the case of K I, all models were computed with a sligthly higher terminal velocity of $15 \mathrm{kms}^{-1}$, as suggested by the blue-shift of the K I line. We note that the red wing of the K I profile is not fully reproduced because of the presence of at least one interstellar component that is not seen in Rb I. 


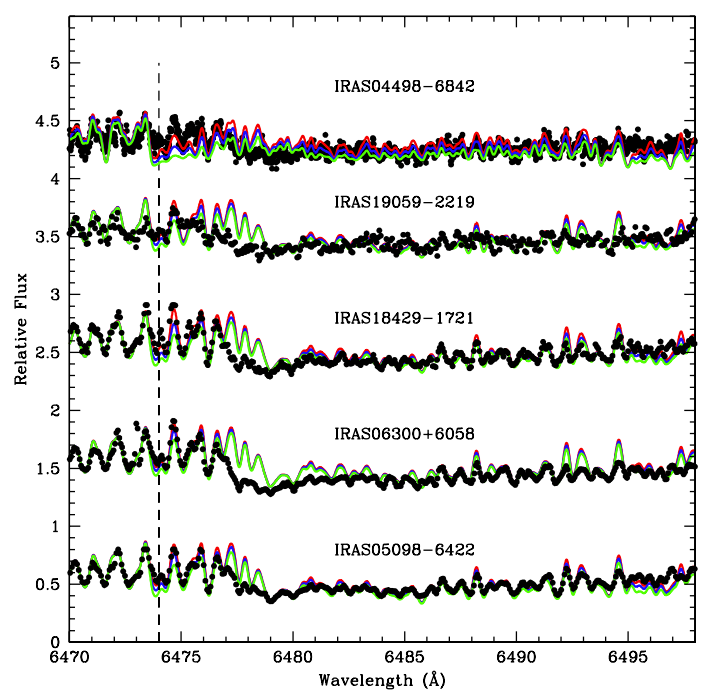

Fig. A.3. High-resolution optical spectra (in black) and dynamical models that provide the best fits (in red) in the $\mathrm{ZrO} 6474 \AA$ A region for our sample stars. Synthetic spectra obtained for $\mathrm{Zr}$ abundances shifted $+0.25 \mathrm{dex}$ (in blue) and +0.50 dex (in green) from the adopted values are also shown.

\section{Appendix B: Monte Carlo simulations}
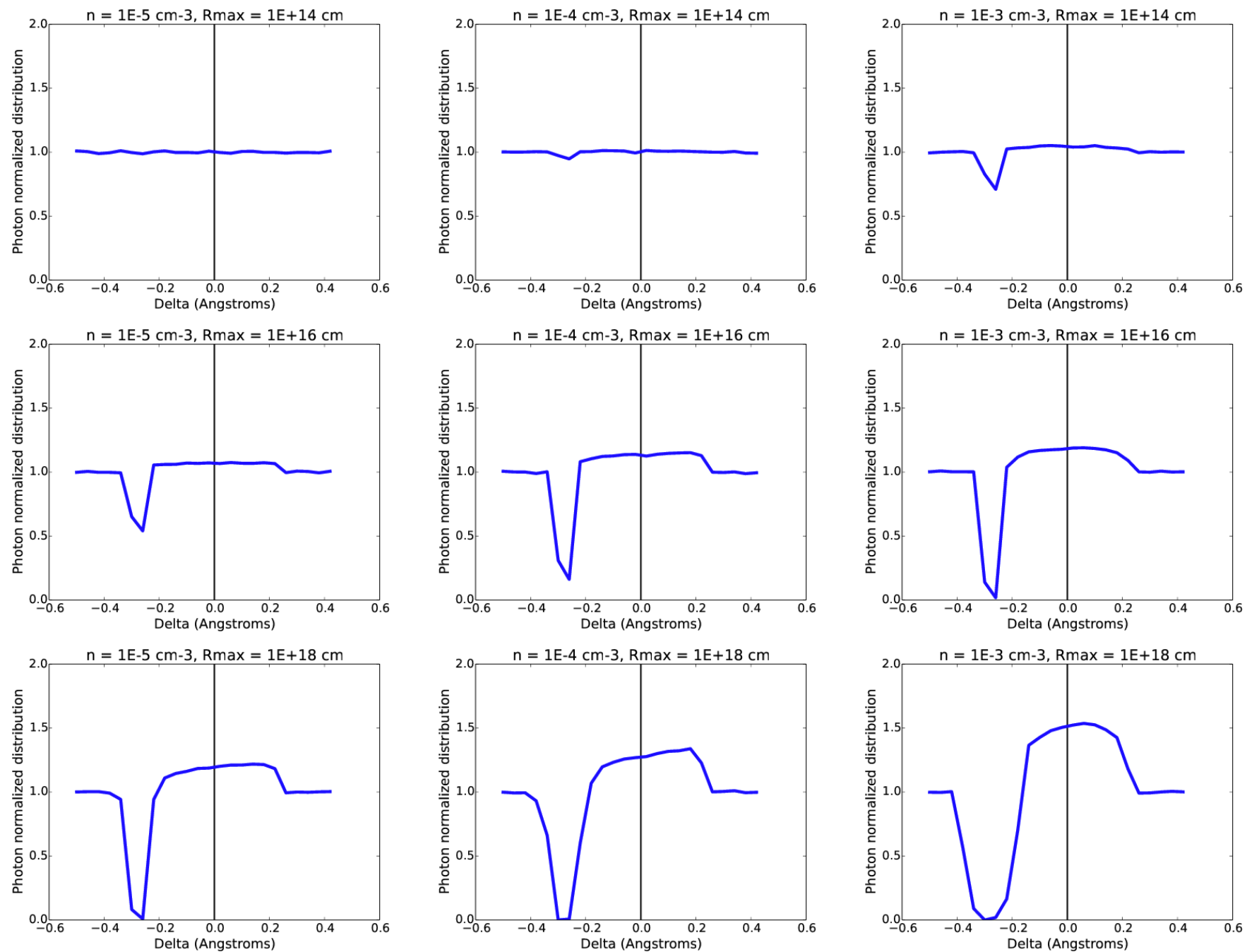

Fig. B.1. Illustrative example of nine Monte Carlo simulations carried out with the envelope parameters $T=1000 \mathrm{~K}$ and $v_{\exp }=10 \mathrm{~km} \mathrm{~s}^{-1}$. The density $\left(n\right.$, in $\left.\mathrm{cm}^{-3}\right)$ and the envelope size $\left(R_{\max }\right.$, in $\left.\mathrm{cm}\right)$ increase from left to right and from top to bottom, respectively. We show the displacement (in Angstroms) from the rest wavelength (Delta $=\lambda-\lambda_{0}$, where $\lambda_{0}$ is indicated by a vertical line) to the emergent wavelength distribution. 